\title{
Recomposition des formats télévisuels de campagne électorale. La Communauté française de Belgique à l'occasion des Élections de 2004
}

Restyling Televisual Campaign Programs: The Belgian French Community during the 2004 elections

\section{Joëlle Desterbecq}

\section{CpenEdition Journals}

Édition électronique

URL : http://journals.openedition.org/questionsdecommunication/403

DOI : 10.4000/questionsdecommunication.403

ISSN : 2259-8901

\section{Éditeur}

Presses universitaires de Lorraine

\section{Édition imprimée}

Date de publication : 1 décembre 2010

Pagination : 197-214

ISBN : 978-2-8143-0056-9

ISSN : $1633-5961$

\section{Référence électronique}

Joëlle Desterbecq, « Recomposition des formats télévisuels de campagne électorale. La Communauté française de Belgique à l'occasion des Élections de 2004 », Questions de communication [En ligne],

18 | 2010, mis en ligne le 01 décembre 2012, consulté le 01 mai 2019. URL : http://

journals.openedition.org/questionsdecommunication/403; DOI : 10.4000/

questionsdecommunication.403 


\title{
$>$ NOTES DE RECHERCHE
}

JOËLLE DESTERBECQ

Observatoire du récit médiatique

Université catholique de Louvain

joelle.desterbecq@uclouvain.be

\section{RECOMPOSITION DES FORMATS TÉLÉVISUELS DE CAMPAGNE ÉLECTORALE. LA COMMUNAUTÉ FRANÇAISE DE BELGIQUE À L'OCCASION DES ÉLECTIONS DE 2004}

\begin{abstract}
Résumé. - Établi sur un territoire plutôt étriqué et perméable aux influences extérieures, le paysage audiovisuel de la Belgique francophone a vu émerger, au cours de la campagne électorale de 2004, deux programmes de facture très différente de ce qui s'était fait jusque-là : «Destination élections » et « Pour qui voter? ». Si l'appartenance de ces programmes au genre politique ne fait pas de doute au vu de leur diffusion au cours d'une période très encadrée légalement et déontologiquement, ceux-ci interrogent néanmoins la dichotomie classique entre formats politiques dits « classiques » et nouveaux formats. En effet, ils s'inscrivent dans un processus global de recomposition des formats politiques télévisuels qui se joue moins sur le plan interne de l'espace d'interaction verbale entre partenaires de la diégèse que sur le plan externe du lien tissé avec le téléspectateur.
\end{abstract}

Mots clés. - Télévision, Belgique, campagne électorale, genres hybrides, nouveaux formats politiques. 
S i de nouveaux rapports se tissent entre politique et médias, si les formes télévisuelles de représentation du politique se transforment, les échéances électorales construisent-elles une temporalité à part entière dans cet univers recomposé de médiatisation du politique? Nous proposons d'aborder cette question au départ de l'examen du paysage audiovisuel de la Communauté française de Belgique tel qu'il s'est présenté au cours de la campagne électorale de 2004. En effet, cette campagne pour les élections régionales et européennes a vu émerger deux programmes de facture très différente de ce qui s'était fait jusque-là : « Destination élections » et « Pour qui voter? ». Tout en s'inscrivant dans le cadre de contraintes afférentes à la période électorale, ces émissions hybrident les genres et surfent sur les changements du mode relationnel entretenu avec le téléspectateur.Après avoir introduit ces programmes et spécifié quelques caractéristiques du paysage audiovisuel belge, nous utiliserons deux méthodologies distinctes : la première prendra pour objet le matériel sémiotique verbal des espaces de débat contenus dans ces programmes. Nous mettrons en œuvre une analyse de discours fondée sur les thèmes et le cadrage des questions. La seconde portera sur le matériel sémiotique audiovisuel. Elle fera référence tant à la sémio-pragmatique de l'image qu'à l'analyse narratologique.

Composée d'une séquence de reportage et de deux séquences d'interview, «Destination élection » est diffusée juste avant le journal télévisé sur la chaîne de service public RTBF durant les deux semaines qui ont précédé l'élection de juin 2004. Après avoir été invité à deviner, via l'utilisation de procédés ludiques, l'endroit où il va se rendre, le candidat aux élections est immergé dans un univers se rapportant aux dossiers dont il est en charge ou aux thèmes de campagne de son parti : assister le travail des puéricultrices dans une crèche; effectuer les démarches d'un demandeur d'emploi; passer une journée avec des jeunes issus de l'immigration; passer une nuit avec les riverains d'un aéroport, etc. Dans l'autocar de la chaîne qui amorce le chemin du retour, le représentant politique est alors conduit à poser un regard rétrospectif sur l'expérience qu'il vient de vivre, à dresser un bilan de la législature qui se termine et à débattre de ses thèmes de campagne. Ainsi, si l'émission est proprement politique, allie-t-elle interview et terrain, use de quizz, de références à des génériques connus Dallas, Un amour de coccinelle - et de renvois à la fiction (la bande magnétique de - Mission impossible et l'iconographie de 24h chrono (Sepulchre, 2005).

«Pour qui voter? » est diffusée en deuxième partie de soirée sur la chaîne privée RTL-TVI au cours de la semaine qui a précédé le scrutin. Cette émission joint au traditionnel débat politique un volet de questionnaire à choix multiples auquel chaque représentant politique présent en plateau est invité à répondre. Une question relative à une thématique électorale est énoncée par le journalisteprésentateur, les candidats doivent alors retrouver, au sein des sous-réponses proposées, la proposition qui fait l'originalité du programme de leur parti. Chaque sous-réponse se voit attribuer un certain nombre de points. Le but du test est double. D'une part, il s'agit d'observer si les scores engrangés par les mandataires 
politiques correspondent bien à ceux qu'ils auraient dû obtenir en retrouvant les propositions électorales de leur parti. D'autre part, le téléspectateur est invité à répondre aux questions et à additionner ses points pour calculer son profil électoral. Parties quizz et débat se succèdent donc dans ce programme qui se clôture par l'évaluation de chaque candidat par le présentateur et le professeur de sciences politiques qui l'assiste.

En France, des recherches ont mis en évidence l'existence d'une polarité entre une temporalité non électorale marquée par un processus de recomposition du politique à la télévision et un temps électoral où l'on en revient à des formats télévisuels somme toute plus classiques (Neveu, 2003; Le Foulgoc, 2003). Qu'en est-il de la Belgique? Le paysage audiovisuel belge possède la particularité d'être câblé depuis le milieu des années 60, mais aussi d'être segmenté politiquement et linguistiquement. Écartelé entre deux télévisions de régime linguistique différent, ce paysage est perméable aux influences extérieures, et spécialement à l'univers médiatique français pour ce qui est de la Belgique francophone (importation de genres, de formules ou de dispositifs d'émissions). En dépit de cette ouverture aux paysages audiovisuels extérieurs, l'étude menée en 2005-2006 par l'Observatoire du récit médiatique de l'Université catholique de Louvain (UCL) sur les programmes auxquels participaient les représentants politiques avait permis de conclure à des transgressions génériques prudentes entre émissions d'information et de divertissement (Antoine, Lits, 2006). Plutôt que d'éviter les échanges politiques, de les marginaliser ou de faciliter le relâchement des modes d'expression, on assistait à une dynamisation formelle de la mise en récit, inhérente à l'évolution du médium télévisuel. Si certaines émissions politiques optent pour une forme qui les tire vers l'univers du jeu ou de la fiction (quizz, questions «formatées », demandes de classement mais aussi infographie, montage dynamique, éclairage, musique et de nombreux renvois intertextuels), dans tous les cas étudiés, celle-ci n'apparaissait jamais comme une fin en soi. Cette conclusion semblait d'ailleurs appuyée par le fait qu'il n'existait pas - il n'existe toujours pas - en Communauté française de Belgique de talk-shows analogues à ceux de Thierry Ardisson ou de Laurent Ruquier en France. Un état de fait qui doit probablement être mis en rapport avec les moyens budgétaires dont disposent les chaînes de télévision dans ces pays.

La question qui nous intéresse ici est de savoir si « Destination élections 》 et «Pour qui voter? » s'inscrivent dans cette dualité entre temporalité électorale et temporalité « usuelle » de présentation du politique en raison du cadre de contraintes qui balise la période électorale. Un cadre fait de dispositions légales et décrétales, d'une part, et de principes issus des usages et pratiques des éditeurs de services, d'autre part. Ou à l'inverse, ces programmes s'inscrivent-ils dans ce processus de dynamisation formelle de la mise en récit, voire poussent-ils plus en avant la transgression des genres en « psychologisant » les questions ou en diluant les thèmes de discussion? Si, comme Érik Neveu l'a montré ( 1991 - 1992; Neveu, Le Grignou, 1993), le dévoilement du personnage privé de l'homme 
politique ne constitue que le fer de lance d'une dilution globale du politique à la télévision, cette psychologisation du politique semble toutefois se faire nettement plus rare en période électorale, où l'on en revient à des formats plus classiques. L'hybridation générique au sein de laquelle s'inscrivent « Destination élections » et « Pour qui voter? » permet-elle une marge de manœuvre?

Afin d'appréhender ces éventuels modes de recomposition, nous reprendrons l'opposition dressée par Patrick Charaudeau et Rodolphe Ghiglione (1999: 27) entre le « contrat interne » d'un programme, présidant à l'interaction verbale qui se déroule dans l'espace filmé, et le « contrat externe » qui relie le téléspectateur aux acteurs médiatiques. En effet, l'analyse de chacune des émissions, séquence par séquence, montre que l'enjeu de la reconfiguration se situe moins sur le plan interne de l'espace d'interaction verbale entre partenaires « homodiégétiques » que sur le plan externe du lien tissé avec le téléspectateur, partenaire « hétérodiégétique » (Genette, 1972 : 255-256). Examinons chacun de ces deux plans.

\section{L'espace d'interaction entre partenaires homodiégétiques}

Pour mener à bien une analyse approfondie, nous avons choisi de travailler exclusivement sur deux émissions de chacun de ces programmes. Nous avons sélectionné deux numéros de « Destination élections 》 aux antipodes du point de vue du degré de mise en danger du représentant politique. Le premier opère l'immersion d'André Antoine, candidat du Centre démocrate humaniste qui fonde sa campagne sur l'importance de l'« Humain » dans une crèche (avril 2006). II participera à toutes les tâches réalisées par les puéricultrices: les jeux, le déjeuner, le goûter, la vaisselle, le couchage des petits. Le second numéro immerge Jean-Claude Van Cauwenberghe, ministre-président wallon et tête de liste du Parti socialiste en Région wallonne/Charleroi, au siège du parti flamand de droite CD\&v (mars 2006). Ne parlant pas néerlandais, il fut confronté au président et aux figures marquantes de ce parti aux revendications communautaires appuyées et qui remportera les élections régionales. Bref, ces représentants politiques ne s'apprécient pas vraiment. Quant aux émissions « Pour qui voter? », elles ont été sélectionnées en fonction du degré de respect de la « règle de jeu ». En effet, le programme est bien structuré en deux parties : le QCM et le débat. Et les mandataires politiques ne sont pas censés débattre dans la section consacrée au quizz. Toutefois, cette règle pose problème en raison du mode de sélection des sous-réponses du QCM par l'instance télévisuelle. Dans les premiers numéros du programme, le journaliste-présentateur laissera une certaine marge de manœuvre par rapport à la règle mais il ne tolèrera plus les « transgressions » dans les dernières émissions. À cet égard, nous avons retenu 
les deuxième et dernier numéros (juillet 2006 et septembre 2006) consacrés respectivement à la Communauté française et à la Région wallonne.

\section{Un espace thématique structuré et ancré dans l'espace public}

Le premier indice qui laisse à penser que la recomposition des programmes se passe moins au plan de l'espace interne réside dans la nature des thèmes développés au sein de ces programmes.

Tableau I : Relevé des thèmes développés dans DE et PQV.

\begin{tabular}{|c|c|c|c|}
\hline \multicolumn{2}{|c|}{ « Destination élections » } & \multicolumn{2}{|c|}{ «Pour qui voter?» } \\
\hline $\begin{array}{c}\text { 03/06 } \\
\text { (Van } \\
\text { Cauwenberghe) }\end{array}$ & $\begin{array}{c}\text { 04/06 } \\
\text { (Antoine) }\end{array}$ & $\begin{array}{c}\text { 07/06 } \\
\text { (Communauté française) }\end{array}$ & $\begin{array}{c}\text { 09/06 } \\
\text { (Région wallonne) }\end{array}$ \\
\hline $\begin{array}{l}\text { - Métalangage } \\
\text { - Politique } \\
\text { intérieure (vie } \\
\text { institutionnelle : } \\
\text { relations } \\
\text { communautaires; } \\
\text { fonctionnement } \\
\text { des entités } \\
\text { fédérées; partis) } \\
\text { - Économique et } \\
\text { social (emploi et } \\
\text { travail; croissance) }\end{array}$ & $\begin{array}{l}\text { - Métalangage } \\
\text { - Famille et vie } \\
\text { sociale } \\
\text { - Finances (budget) } \\
\text { - Éducation, } \\
\text { enseignement } \\
\text { - Politique } \\
\text { intérieure (vie } \\
\text { institutionnelle : } \\
\text { fonctionnement } \\
\text { des entités } \\
\text { fédérées; partis) } \\
\text { - Économique et } \\
\text { social (entreprises } \\
\text { et investissements) }\end{array}$ & $\begin{array}{l}\text { - Éducation, } \\
\text { enseignement } \\
\text { - Finances (budget, } \\
\text { financement) } \\
\text { - Culture et industries } \\
\text { culturelles (statut des } \\
\text { artistes, développement } \\
\text { culturel, musées, } \\
\text { création) } \\
\text { - Politique intérieure (vie } \\
\text { institutionnelle: } \\
\text { fonctionnement des } \\
\text { entités fédérées) } \\
\text { - Sport (pratique } \\
\text { sportive, sport et } \\
\text { handicap, sport de haut } \\
\text { niveau, sport-études) }\end{array}$ & $\begin{array}{l}\text { - Finances (fiscalité) } \\
\text { - Économique et } \\
\text { social (entreprises, } \\
\text { investissement et } \\
\text { exportations; emploi et } \\
\text { chômage; logement) } \\
\text { - Environnement } \\
\text { (aménagement du } \\
\text { territoire) }\end{array}$ \\
\hline
\end{tabular}

Ce ne sont pas des faits de société qui sont débattus, mais bien des thématiques caractérisées par leur « ancrage dans l'espace public à travers les différents domaines de représentation de la pratique sociale qui le construisent » (Charaudeau, Ghiglione, 1997 : 78). En effet, les champs politiques, économiques, sociaux et culturels constituent les fondements des échanges verbaux. Notons, au sein des thématiques de Destination élections, un item intitulé : « métalangage ». Celui-ci se rapporte à la première séquence conversationnelle, celle qui a pour 
but de faire deviner le lieu de destination. On y traite du « jeu » lui-même, on découvre les destinations qui plairaient ou déplairaient au candidat. II s'agit de la seule thématique qui sort des domaines de représentation politiques, économiques, sociaux ou culturels des séquences conversationnelles. Néanmoins, cette thématique ne dépasse pas trois minutes par émission. Elle est donc assez mineure dans ce programme de 35 minutes.

Par ailleurs, dans le programme de la RTBF comme dans celui de RTL-TVI, les foyers thématiques sont définis en priorité par l'instance médiatique, ce qui contribue à structurer l'espace de thématisation. Deux dimensions concourent à son organisation. D'abord, les deux émissions sont structurées par un macro-thème. Celui-ci va de pair avec l'immersion que vivra le représentant politique dans « Destination élections ». II correspond, en outre, aux « entités » pour lesquelles l'électeur sera amené à voter dans « Pour qui voter? ». Cette définition d'un macro-thème délimite un premier niveau de structuration qui ne constitue pas forcément une règle dans les programmes électoraux - une comparaison mériterait d'être établie notamment avec les émissions fondées en priorité sur l'agenda citoyen. Ensuite, ce macro-thème fait l'objet d'une division linéaire en chapitres (RTL-TVI) ou en thèmes premiers et périphériques (RTBF). Certes, le représentant politique dispose d'une certaine latitude en vue d'introduire des sous-thèmes, mais ils sont assez rapidement recadrés ou balayés par l'introduction d'autres thématiques « médiacentrées ».

\section{Un cadrage politique}

Se borner à énoncer les thématiques développées dans les échanges ne semble toutefois pas suffisant afin d'évaluer la finalité des propos: tout dépend de l'orientation de la question de l'instance d'animation. En effet, un thème « famille et vie sociale », tel qu'initié dans l'émission « Destination élections » d'avril 2006, peut être développé sous l'angle du récit de vie familial ou de la parole d'engagement politique. Pour dissocier ces finalités conversationnelles, nous proposons d'étudier le cadrage ( « framing ») des questions en termes de contenu. "Framing involves « selecting and highlighting some facets of events and issues, and making connections among them so as to promote a particular interpretation, evaluation, and/or solution » »' (Entman, 2006 : | 34). Ainsi, selon que l'instance d'animation sélectionne telle ou telle « facette » des événements, ses questions porteront-elles sur différentes finalités. Nous en distinguerons trois: les questions adressées au locuteur politique peuvent porter sur des politiques publiques (actions ou projets) et/ou sur le positionnement du mandataire dans le jeu politique, mais elles peuvent porter aussi sur sa personne, voire sur le discours

Traduction: « Cadrer [des événements] implique de sélectionner et de mettre en évidence certains aspects des évènements et des enjeux, et d'établir des liens entre ceux-ci, afin de favoriser une certaine interprétation, une évaluation et/ou une solution ». 
médiatique lui-même. Nous identifierons le premier type de questions par le terme de « cadrage politique », le deuxième par « cadrage personnel » et le troisième par « méta-cadrage ». Le « cadrage politique » est traditionnellement scindé en deux types de contenus désignés par une pluralité de concepts qui recouvrent toutefois des distinctions assez semblables : jeux et enjeux (Gerstlé, Duhamel, Davis, 1992), substantive et procedural frames (Entman, 2004) ou encore issue-specific et generic frames (Callaghan, Schnell, 2005). Si ces deux types de cadres se chevauchent fréquemment, nous tenterons d'en repérer le « centre de gravité ou noyau thématique » (Gerstlé, Duhamel, Davis, 1992 : 54). Par ailleurs, le « cadrage personnel » recouvre des questions portant sur le récit de vie d'un individu (la narration du quotidien ou la restitution de traces de sa mémoire), sur des données biographiques ou encore sur ses passions, goûts et sentiments. Enfin, dans le « méta-cadrage », les médias deviennent leurs propres objets de discours. Par conséquent, les questions adressées au mandataire politique porteront sur le programme lui-même ou sur ses impressions par rapport au dispositif.

Nous avons additionné les questions et demandes de validation adressées aux représentants politiques des deux numéros de « Destination élections », d'une part, et de « Pour qui voter? », d'autre part. Les moyennes calculées pour chaque programme laissent entrevoir deux modes de cadrage très différents.

Graphique I : Cadrage des questions en termes de contenu.

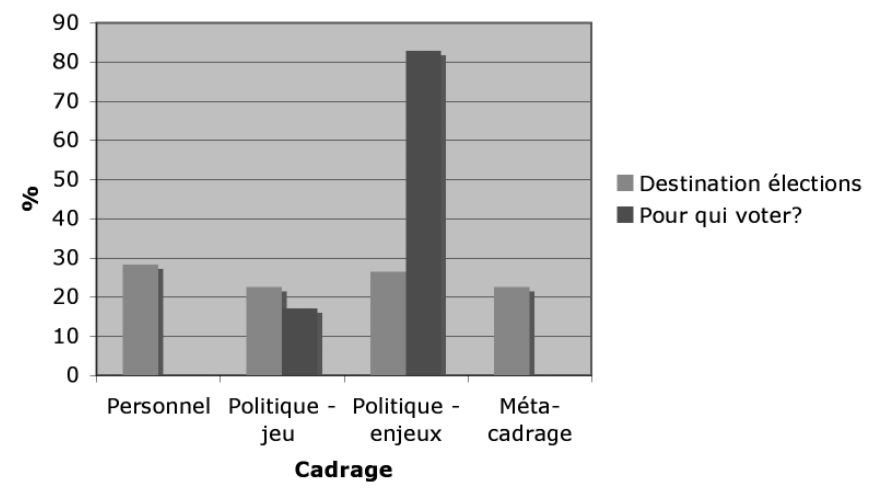

Le premier constat renvoie au cadrage proposé dans « Pour qui voter? » : les séquences conversationnelles de ce programme de RTL-TVI portent exclusivement sur des contenus proprement politiques. C'est au départ des thèmes des QCM de la section ludique que sont construites les séquences conversationnelles de débat entre mandataires politiques. Or, ces QCM sont élaborés sur la base des propositions d'actions politiques contenues dans les manifestes de partis. Aussi la section ludique du programme, recourant aux outils des jeux télévisés, n'atténue- 
t-elle paradoxalement pas la substance politique de l'émission, mais elle semble la renforcer. Qui plus est, en regard de la tendance de nombreuses émissions électorales à hypertrophier les sujets consacrés au « jeu 》 ou aux stratégies politiques (Gerstlé, Duhamel, Davis, 1992; Strömback, Dimitrova, 2006), « Pour qui voter? » se focalise à plus de $80 \%$ sur les enjeux, les questions de politiques publiques. Par exemple: «Anne-Marie Corbisier, quel est le remède principal à mettre en œuvre pour essayer d'améliorer la qualité de l'enseignement? » (juillet 2006); « Alors, l'emploi et l'économie. Serge Kubla, on arrive à attirer des investisseurs? Ou, en tout cas, davantage d'investisseurs que dans le passé? » (septembre 2006).

En référence aux indicateurs de Jacques Gerstlé et d'Érik Neveu, les questions que nous avons regroupées sous l'intitulé de stratégies ou « jeu » politique portent sur les attaques, les soutiens, les réactions, les évaluations, les affaires, la campagne, l'image, les sondages (Gerstlé, Duhamel, Davis, 1992 :54) mais aussi sur les alliances, les positionnements face aux rivaux, les luttes internes, les tactiques, les polémiques et les attributions de postes (Neveu, 1997 : 36). En revanche, ces questions atteignent une moyenne de près de $23 \%$ dans l'émission de la RTBF (soit presque autant que les enjeux politiques) qui ne fonde pas ses séquences conversationnelles sur les manifestes de partis mais bien sur les dossiers - dont est en charge le mandataire politique - ou sur les thèmes de campagne de son parti. C'est surtout la question des alliances, des soutiens et des positionnements face aux rivaux qui domine dans les deux numéros étudiés: «On est face à une période très, très importante pour eux [le CD\&V] C'est important de vous donner une bonne image. Vous avez l'impression que politiquement parlant, il y a un enjeu là derrière? » (mars 2006); « 《ous êtes surtout critique à l'égard du Mr. Le CDH a l'air plus humaniste vis-à-vis du PS et d'ÉCOLO » (avril 2006).

Le second constat majeur de cet examen du cadrage des questions en termes de contenu se rapporte à la diversité des modes de cadrage figurant dans «Destination élections ». Si la moitié des questions de l'émission du service public porte sur un cadrage politique (jeux et enjeux), l'autre moitié se répartit entre des interrogations prospectives sur le terrain à venir (ou rétrospectives sur l'expérience que lui a donnée à vivre le média) et des interrogations sur la personne du candidat en campagne. II s'agit là d'un trait distinctif de « Destination élections ». Toutefois, pour mesurer l'importance de ce cadrage a priori psychologisant, déployé au cours de la période très réglementée de la campagne électorale, il convient d'observer le rôle exercé par ces questions dans l'organisation de l'échange. Nous avons donc re-catégorisé les questions et demandes de validations, non plus au départ de leur contenu mais en prenant en considération le rôle qu'elles exercent dans la progression thématique de l'échange (initier l'échange, relancer, réparer une mécompréhension, réguler, alterner et ritualiser). Au départ de cette catégorisation, il s'est avéré que plus de la moitié des questions personnelles adressées dans les deux numéros étudiés relèvent d'un mode de questionnement rituel. Récurrentes dans les différents numéros 
du programme, ces questions exercent une fonction moins transactionnelle de transmission d'informations, que relationnelle d'introduction ou de clôture de l'interaction. Ce sont donc des questions essentiellement phatiques, permettant l'entrée ou la sortie de l'échange. « Où passeriez-vous volontiers une journée et avec qui? »; « Quel est l'endroit où vous aimeriez aller? »; « Une journée de cauchemar, ce serait avec qui et où? »; « Vous vous sentiriez piégé si on vous emmenait où? » constituent quelques exemples de ces interrogations portant sur les goûts. Elles agissent comme une mise en contexte, tendent à capter le téléspectateur qui découvrira l'aisance ou le malaise du représentant politique une fois in situ, bien plus qu'à cerner réellement le contour de la personnalité du représentant politique. D'autant que les réponses des candidats sont, le plus souvent, politiques. Par exemple : « J'aurais été à Châtelet à Carinox, là où ARCELOR va investir 10 milliards d'anciens francs 》 (DE, mars 2006). Les qualités et goûts personnels du candidat sont esquissés à des fins d'accroche mais on ne peut prétendre que le programme érige cette dimension en paramètre de choix électoral, d'autant que certains choix de terrain se prêtent mieux que d'autres à l'ébauche de ces questions. Certaines questions, plus personnalisées, introduisent par ailleurs des thèmes de discussion. Par exemple, en interrogeant André Antoine sur son rôle de père - «Voilà une question qui pourrait vous mettre un peu sur la voie: vous êtes papa, vous avez trois enfants. lls ont quel âge? »; «Vous aviez fait ça à l'époque ou bien tout ça c'était pour madame le travail de langes, panade? » (avril 2006) -, la journaliste part du particulier pour introduire la problématique générale des mesures en faveur de la petite enfance, fer de lance de son parti. Ces questions sont davantage des techniques d'accroche que des finalités réelles dans les séquences discursives du programme. C'est donc essentiellement sur la fonction phatique de contact avec le téléspectateur que ces programmes semblent innover, plutôt que sur le fond des échanges.

\section{L'espace d'interaction entre partenaires homodiégétiques et hétérodiégétiques}

Ainsi, si nous avons été amenés à penser que la recomposition des émissions «Destination élections » et « Pour qui voter? » se passe moins au plan de l'espace interne, c'est parce que la psychologisation des questions n'est pas systématique sous la forme ludique des programmes - ou qu'elle ne constitue pas une fin en soi - mais aussi parce que ces émissions ne diluent pas les thèmes de discussion, pas plus qu'elles n'éclatent l'espace thématique. En revanche, il semble que ces programmes électoraux s'inscrivent dans une évolution générale de la grammaire télévisuelle du point de vue du « contrat externe » tissé avec le téléspectateur. Ils intègrent et surfent sur les changements « du mode relationnel » (Lochard, Soulages, $1998: 67)$ noué avec le téléspectateur. 


\section{«Destination élections » : participation spectatorielle et « télévision relationnelle »}

Afin de renforcer le lien avec le téléspectateur, « Destination élections » joue à la fois sur les dimensions classiques de la participation spectatorielle et importe certaines composantes de la « télévision relationnelle » (Mehl, 2002) et de son avatar le plus récent, la télé-réalité. Comme le rappelle Jean-Pierre Meunier (2004: 177), « à l'état spectatoriel, furent associées les notions de participation et d'identification : participation du spectateur au déroulement du film, identification aux personnages 》. « Destination élections » use de plusieurs ressorts filmiques afin de favoriser cette adhésion au perçu ou cette plongée dans l'univers de la diégèse, le monde de l'image.

Le premier d'entre eux n'est autre que le montage dont l'amplitude narrative forte favorise l'entrée en résonance avec le cours des événements qui se déroulent sur l'écran. Nous reprendrons la typologie des agencements séquentiels de Christian Metz (197I) afin d'étudier le montage des séquences « reportage » qui immergent les représentants politiques sur le terrain. En prenant en compte les critères de contiguïté ou de simultanéité non seulement spatiale mais aussi temporelle, cette typologie offre la possibilité de cerner les visées narratives du récit filmique. «Destination élections » suit une construction entièrement fondée sur des syntagmes chronologiques, dans la mesure où le rapport temporel entre les faits est nettement précisé. En effet, l'infographie propose un écoulement du temps en reprenant le dispositif de la série 24 heures chrono. Au départ de cette armature chronologique, l'émission fait se succéder syntagmes narratifs alternés et syntagmes narratifs linéaires. Le premier type est fondé sur deux séries d'événements qui se déroulent simultanément, le second prend pour base une seule série d'événements marqués par un rapport de consécution temporelle. En effet, le reportage propose plusieurs split screens qui présentent des événements se déroulant simultanément dans des lieux distincts. Ces split screens marquent toujours une transition vers un nouveau terrain. Les événements du terrain sont alors portés à l'écran au départ de « séquences ordinaires », modalité du syntagme narratif linéaire dans lequel le montage « saute » certains moments pour des raisons fonctionnelles. Cette continuité narrative découlant du montage favoriserait l'immersion dans la diégèse, la participation spectatorielle. En effet, si on se réfère à Muriel Hanot (2002: II-1 I7) étudiant les effets de réalité des émissions de télévision, la succession spatio-temporelle et l'enchaînement logique des différents syntagmes chronologiques permettent de construire une continuité narrative qui sollicite participation et syncrétisme de la part du récepteur.

Le deuxième ressort filmique dont use « Destination élections » en vue de favoriser l'intégration dans le monde diégétique relève de l'articulation entre le son et l'image. En effet, la bande son du reportage ne comporte aucune « voix over », voix d'un énonciateur hors diégèse dont le propos viendrait recadrer la 
mise en images. Par ailleurs, les personnages du monde de l'image ne s'adressent jamais directement au spectateur par l'intermédiaire d'une intervention en axe $y-y$, par exemple. Le dispositif d'énonciation du reportage ne comporte donc pas de « rupture de diégèse », pour reprendre un terme développé par les analyses cinématographiques. «L'adresse au spectateur a quelque chose de tout à fait paradoxal. Celui qui s'adresse au spectateur établit un contact en fait impossible entre le monde imaginaire - d'où il s'adresse au spectateur - et le monde réel où se trouve celui-ci -, et c'est de cette mise en contact que surgit l'évidence de leur séparation » (Meunier, Peraya, 2004 : 244). L'absence de marques d'adresse au spectateur favorise donc l'abandon dans l'image, la participation au perçu. Enfin, « Destination élections » encourage la participation spectatorielle en jouant quelque peu sur les ressorts du suspense. En effet, au cours des premières minutes du reportage, le téléspectateur découvre la destination avant le personnage politique. Le programme cherche donc à développer le suspense en travaillant sur le mécanisme de «focalisation » (Genette, 1972 : 206). La quantité d'informations donnée au spectateur comparativement à celle dont dispose le personnage est une manière de régir le mécanisme de suspense narratif. Comme l'ont mis en évidence les travaux de François Jost sur la focalisation au cinéma, la « structure de focalisation zéro ou spectatorielle, c'est-à-dire une structure où l'on annonce au spectateur un danger que le personnage ignore, le spectateur jouissant alors d'un savoir qui excède celui du personnage » est un mécanisme générateur de suspens. L'émission joue ensuite sur l'alternance des points de vue en passant de la focalisation zéro à la focalisation interne dans laquelle le récit fait connaître les événements tels qu'ils se présentent au personnage. C'est son ressenti que l'on donne à voir dans la suite du reportage.

Si le programme électoral de la RTBF sollicite l'état spectatoriel, il emploie également certains mécanismes de la « télévision relationnelle 》 décrits par Dominique Mehl (2002 : 93) :

« La télévision relationnelle confie à l'écran le soin de créer une relation, relation entre artistes et public, entre responsables politiques et citoyens, entre journalistes ou animateurs et téléspectateurs, entre studios et salons, et les liens tissés comptent plus que les propos tenus. Elle joue sur la partition de la convivialité et l'individu se trouve au centre du dispositif [...] Cette télévision est impulsée par la demande et l'écran devient un miroir censé refléter les désirs, les goûts, les habitudes, les modes de vie, les valeurs, les préoccupations du public ».

« Destination élections » importe de cette télévision miroir l'intégration de l'individu à l'écran. Cette émission propose une nouvelle étape d'incorporation du citoyen ordinaire, sans référence particulière dans la scène télévisuelle électorale. À l'exception de l'émission consacrée à une immersion au siège du parti CD\&V, le programme donne à voir « Monsieur tout le Monde » confronté aux décisions des représentants politiques : les puéricultrices face au manque de places dans les crèches et au manque de subventions; les riverains d'un aéroport survolés par les vols de nuits; les jeunes d'une maison de quartier aux prises avec la conception de l'ordre public de leur maire, etc. C'est le vécu par les citoyens ordinaires des décisions de la classe politique que vise le concept de l'émission. 
L'individu ordinaire est donc passé à un stade supérieur de participation à un programme électoral :il fut public passif,puis public plus ou moins réactif, disposant du droit de s'adresser directement au représentant politique. II est maintenant un personnage à part entière de la scène télévisuelle dont on expérimente la quotidienneté. Nous sommes conscients que le représentant politique, et non l'individu lambda, constitue le noyau du dispositif de "Destination élections ». Toutefois, ce que nous visons est bien l'intégration d'un mode de saisie de la réalité généralisé par la real TV dans une sphère spécifique de la programmation politique au sens strict.

De la même manière, on retrouve dans «Destination élections 》 trois ingrédients majeurs caractérisant les espaces filmiques de la télévision relationnelle et de la télé-réalité - cet apogée de la néo-télévision de relation : le « cadre contraint », «l'expérimentation de la quotidienneté » et la « gestion des relations humaines » (Antoine, 2004 : 27-32). « Les projecteurs sont braqués sur les relations interindividuelles qui se nouent et se dénouent entre des personnes appelées à mettre en jeu leur personnalité dans des scènes domestiques et familières artificiellement créées par le média », stipule Dominique Mehl (2008 : 269) pour esquisser le visage de la télévision relationnelle. C'est une mise au jour identique de la compétence relationnelle des mandataires publics que vise l'émission de la RTBF. « Dans « Destination élections », l'objectif était, quelque part, de confronter l'homme politique à des situations qu'il est en principe amené à traiter, et donc de voir quelle attitude il adopte dans ces situations-là », précise l'un des concepteurs du programme (interview avec les éditeurs de services in : Antoine, Lits, 2006). Les éditeurs de services semblent donc se pencher sur la monstration des relations interpersonnelles, à l'instar des nombreux programmes de la real $T V$ et de leur « catalogue des comportements humains » (Antoine, $2004: 32$ ). Par ailleurs, si l'espace d'interaction de cette dernière se veut domestique et familier, « Destination élections », elle, cherche sans doute moins la domesticité que la familiarité : l'habituel, les gestes connus, les situations du quotidien (trouver une place de crèche, dormir, prendre les transports en commun, etc.). Cette confrontation du politique par rapport à une quotidienneté est d'ailleurs revendiquée par les concepteurs du programme :

«La politique n'est pas pour moi quelque chose de conceptuel, de décalé par rapport à la vie
quotidienne. La politique c'est du concret, c'est pas des choses qui échappent aux gens [...]. Les
hommes politiques sont là pour s'impliquer dans des dossiers qui concernent tout le monde. Et
c'est ça l'idée d'amener les gens sur le terrain, de les amener à la rencontre des acteurs de la vie
quotidienne » (interview avec les éditeurs de services in : Antoine, Lits, 2006).

Les caméras captent donc les attitudes ou les rapports inter individuels dans des situations de terrain quotidiennes. Mais, comme dans l'espace filmique de la TV relationnelle, ces mises à l'épreuve interpersonnelles sont créées de toutes pièces par le média lui-même. Les lieux ne sont pas inventés mais l'espace d'interaction est provoqué : «Tout est scénarisé entre guillemets. On décide du choix du lieu, on a pris contact avec la personne, j'ai fait moi-même des repérages dans les lieux [...]. Tout est réel mais tout est scénarisé, c'est-à-dire qu'à chaque 
moment, à chaque porte qu'on passait, je savais où on allait et je savais qui on allait rencontrer » (interview avec les éditeurs de services in: Lits, 2006). «Destination élections » renvoie donc à un univers quotidien, provoqué par le média. On y entrevoit l'importation de composantes de captation constitutives des programmes de real TV, témoignant en cela du rôle de « filtre réformateur » que joue la télé-réalité vis-à-vis de l'ensemble du média télévision (ibid. : 72), y compris dans ses sphères réservées au journalisme politique.

\section{«Pour qui voter?? : pédagogie ou captation}

Le dispositif de « Pour qui voter? » semble vouloir concilier captation et pédagogie en proposant aux téléspectateurs un aperçu des programmes des partis politiques sous la forme de réponses à un questionnaire à choix multiples. II y aurait « pédagogie » puisque l'objectif est de proposer aux téléspectateurs de dresser leur profil électoral. II y a donc une dimension d'enseignement quant au contenu des manifestes de partis, un projet de partage de connaissances appuyé par la figure de l'expert-politologue. II y aurait, par ailleurs, « captation » au vu de la forme du programme. Celui-ci fut annoncé dans un hebdomadaire de programmes TV en tant que « jeu questionnaire qui confronte les candidats et les téléspectateurs à une série de questions dont les réponses révéleront les sensibilités politiques » (Le Soir Magazine, in : Lits A., 2006, vol. d'annexes). Toutefois, l'ensemble de l'émission paraît marqué par des difficultés quant à la concrétisation ou conciliation de ces finalités.

Penchons-nous sur la finalité de captation. Après l'énonciation d'une thématique électorale, les représentants politiques doivent retrouver la proposition qui fait l'originalité du programme de leur parti. Chaque sous-réponse se voit attribuer un certain nombre de points. Le total des points renvoie à une sensibilité électorale particulière. La section questionnaire du programme s'inscrit donc dans le «mode d'énonciation ludique » : elle parle du « monde » réel tout en se conformant à des règles propres. Comme l'a rappelé Jean-Pierre Esquenazi (200I : 5 I-52) à la suite de François Jost, « les règles rigoureuses des mondes ludiques imposent des contraintes fortes aux discours qui leur correspondent. Ceux qui jouent n'ont guère de choix : ils doivent utiliser les énoncés que le jeu laisse à leur disposition. Ils doivent devenir des joueurs, c'est-à-dire des utilisateurs d'un vocabulaire et d'une syntaxe qui leur préexistent ». Ainsi, dans le monde ludique de « Pour qui voter? », la sphère d'action politique est-elle réduite aux conditions de décidabilité du QCM (une seule proposition par parti). En revanche, dans la section débat du programme, le « joueur » est invité à retrouver son identité initiale, c'est-à-dire à proposer au citoyen un projet de société et à persuader du bien-fondé de ses propositions dans un espace d'échanges contradictoires. Toutefois, ces alternances voulues par l'instance télévisuelle troublent fréquemment le bon déroulement du programme. En effet, l'émission est marquée soit par une mécompréhension des règles du jeu, soit par une remise en cause des règles, soit par les deux. Plus 
spécifiquement, c'est la parcellisation du savoir politique, telle qu'elle est initiée par la règle, qui est mise en cause. Elle donnera lieu à de nombreuses interventions de cadrage dans la section ludique de questionnaire: " Sur ce thème, quand on regarde $A, C$ et $D$, je pense que vous les retrouverez à peu près dans tous les programmes » (juillet 2006); « Je voudrais faire un commentaire parce que c'est simpliste... » (septembre 2006). Plusieurs représentants politiques ont souligné la difficulté de concilier les conditions de décidabilité du QCM, qui imposent une réduction du patron de sous-réponses, avec les caractéristiques intrinsèques d'un système politique multi-partisan recourant aux coalitions: « Pour chaque question posée, ils avaient retenu un élément du programme des partis politiques, alors qu'on pouvait se retrouver dans beaucoup d'autres. On sait que les programmes des partis s'interpénètrent, que souvent, de moins en moins d'éléments nous séparent », précise l'un des participants (interview avec les représentants politiques, in : Antoine, Lits, 2006).

La section questionnaire de «Pour qui voter? » inscrit donc le programme à la croisée de deux chemins. D'une part, il fait partie de ces nouveaux formats politiques qui effacent les barrières entre les mondes authentifiants, fictifs et ludiques (Jost, 1999). II sollicite entre ses sections conversationnelles et ludiques les « changements d'identité discursive » mis en évidence par Jean-Pierre Esquenazi (200I : 52) : « Il est possible d'endosser l'identité d'un joueur, de créer un personnage puis de revenir à l'une de ses identités réelles habituelles dans une durée réduite ». D'autre part, l'émission vise à faire partager des connaissances politiques au départ de contenus pris dans leur forme la plus rigide : il s'agit d'extraits de manifestes de partis. En véhiculant ce type de connaissances, elle s'inscrit dans un horizon d'attentes semble-t-il fort peu malléable.

Ces difficultés rejaillissent sans conteste sur sa visée « pédagogique ». En projetant dans la section ludique les caractéristiques intrinsèques du QCM d'examen sur le débat politique, on aboutit à une évaluation du représentant politique. Cette métaphore est d'ailleurs récurrente dans les échanges verbaux des différentes émissions : «En studio, c'est l'heure de vérité. Il y a eu quelques erreurs commises tout au long du parcours... » (présentateur, septembre 2006); « Olivier Chastel, un peu plus à gauche que le MR, à un point près. Pour le CDH, Anne-Marie Corbisier, c'est tout juste mais ça va encore. Jean-Marc Nollet, vous êtes bien Écolo; Christian Dupont, vous êtes bien socialiste. L'examen est globalement réussi » (présentateur, juillet 2006). On évalue la performance «bonne » ou « mauvaise » d'un individu plutôt qu'on ne se fixe sur le contenu du manifeste de parti au départ duquel il y a évaluation. La difficulté de conciliation des finalités pédagogique et de captation prend ici toute son ampleur. « Pour qui voter? » travaille sur le contrat externe du lien tissé avec le téléspectateur en proposant un ancrage ludique plus attractif dans la forme. Toutefois, cet ancrage rejaillit sur la finalité du programme : l'apprentissage d'un contenu, même partiel, schématique et renvoyant un tant soit peu à nos catégories pourrait être contourné par la focalisation sur le sujet et sa performance, bonne ou médiocre. 


\section{Conclusion}

S'il existe un cadre de contraintes avec lequel les éditeurs de services doivent composer au cours des semaines précédant les échéances électorales, ce cadre ne prévient pas toute inscription des émissions électorales dans une dynamique générale de recomposition des formats politiques télévisuels. Toutefois, l'analyse des émissions de la campagne 2004 révèle que cette recomposition se joue moins sur le plan du « contrat interne », qui préside aux échanges entre partenaires intra-diégétiques, que sur le plan du « contrat externe » visant le lien tissé avec le téléspectateur. En effet, dans « Pour qui voter? », le cadrage des questions demeure exclusivement politique. Et si « Destination élections » tend à personnaliser un tant soit peu les questions, ce mode de cadrage ne constitue jamais une finalité en soi. Les deux programmes ne présentent, par ailleurs, aucune dilution des thèmes de discussion ou d'éclatement de l'espace thématique. En revanche, ces émissions surfent sur les changements du mode relationnel entretenu avec le téléspectateur propre à la néo-télévision. À cette fin, soit elles jouent sur les dimensions classiques de la participation spectatorielle et sur plusieurs ressorts de la télévision de relation, soit elles sollicitent des « changements d'identité discursive » auprès de leurs participants. « Destination élections » et « Pour qui voter? » interrogent donc la dichotomie classique entre formats politiques dits « classiques » et nouveaux formats. II importe toutefois de préciser que ces deux programmes constituent une offre parmi d'autres au sein des émissions consacrées à la campagne électorale de 2004. En effet, toutes deux prennent place au sein d'un panel de programmes de facture sans doute plus « traditionnelle », c'est-à-dire moins axée sur le projet de captation du téléspectateur. Ce sont des face-à-face et des débats électoraux. II semble donc que ces émissions électorales « recomposées 》 ne constituent qu'une offre complémentaire dans les dispositifs télévisuels de campagne plutôt qu'une offre de substitution. Néanmoins, l'émergence de ces nouveaux formats électoraux, qui exacerbent la dimension relationnelle, aux côtés des programmes de débat de facture « traditionnelle » renvoie à la question du « morcellement » dont l'espace public ferait actuellement l'objet. Cette position épistémologique, que I'on retrouve dans les travaux de Bernard Miège dès 1989, de Bastien François et Érik Neveu (1999), ou encore de Mihai Coman (2003), met en exergue la fragmentation structurelle d'un espace public de plus en plus hétérogène en « espaces partiels » qui se juxtaposent ou s'interconnectent. À cet égard, la contribution de Mihai Coman à la notion d'espace public intéresse au premier chef l'analyse de la recomposition des formats de campagne électorale. Cet auteur postule que l'espace public et son médium, l'usage public de la raison, ne seraient plus uniquement liés à l'argumentation, mais qu'il existerait aussi des rationalités rituelles, symboliques et narratives : « La mise en forme narrative et le débat dans les termes du langage symbolique n'excluent ni la rationalité, ni le bon sens, ni la chance d'atteindre une « vérité » assumée en commun » (Coman, 2003 : 179). L'auteur conclut donc non seulement à l'existence d'un « espace public symbolique », mais aussi à celle d'un espace public global non homogène. 
En reprenant l'expression de « public sphericules » proposée par Todd Gitlin, il invite à distinguer plusieurs espaces publics « locaux» qui se croisent et parfois s'affrontent au sein d'un espace public généralisé (ibid. : 179). Ces émissions électorales, qui associent au contenu « politique » une dimension relationnelle axée sur l'immersion du téléspectateur dans la diégèse, la gestion des relations humaines ou l'alternance entre les identités discursives de joueur et de débattant, ne renverraient-elles pas à ces rationalités narratives ou symboliques? La confrontation des points de vue se déroule certes selon des processus différents de ceux consacrés par la modernité et les personnalités entrent inévitablement en compte, mais est-ce pour autant que l'on exclut les opinions ou l'idée même de débat? La question est ouverte. La coexistence de ces formules aux côtés des programmes dits politiques au sens «strict » laisse à penser à un éclatement de l'espace public entre des espaces axés sur une rationalité symbolique et d'autres fondés sur l'argumentation. Cet éclatement prévaut sans doute aussi du côté de la réception car le téléspectateur qui choisit de s'exposer à l'espace symbolique n'est probablement pas celui qui fait le choix des formats classiques de débat. Quant au représentant politique soucieux de toucher un électorat élargi, il peut passer d'un format à l'autre ou d'une « public sphericule » à l'autre, sédimentant par là ses registres de parole et son identité socialement construite. Une remarque s'impose encore pour attester définitivement d'une recomposition globale des formats politiques électoraux en Communauté française de Belgique. II conviendrait de mener cette analyse dans une perspective diachronique en observant la récurrence de tels formats « hybridés 》. À cet égard, les formats des campagnes électorales de 2007 (élections législatives) et de 2009 (élections régionales et européennes) se sont tous avérés beaucoup plus conventionnels, mettant de côté les séquences de reportage in situ, les ressorts fictionnels ou ludiques. En revanche, ils ont proposé, sur les deux chaînes privée et publique, des dispositifs interactifs donnant corps à la parole citoyenne. Il conviendrait donc d'élargir les perspectives en étudiant ces changements du mode relationnel sur une plus longue période et en observant si, in fine, ils modifient la configuration de l'espace public global.

\section{Références}

Antoine F., 2004, « La télé-réalité : un nouveau genre télévisuel? 》, pp. 19-81, in: Wangermée R., dir., À l'école de la télé-réalité, Bruxelles, Éd. Labor.

Antoine F., Lits M., dirs, 2006, La spectacularisation des personnalités politiques dans les programmes de télévision de la Communauté française, Rapport de recherche pour le CSA et le Parlement de la Communauté française, 2 vol.

Charaudeau P., Ghiglione R., 1999, Paroles en images. Images de paroles. Trois talk-shows européens, Paris, Didier Érudition.

— 1997, La parole confisquée. Un genre télévisuel : le talk-show, Paris, Dunod. 
Recomposition des formats télévisuels de campagne électorale

Chateauvert J., 1994, « Les mécanismes », CinémAction, 71, pp. |36-|4 |.

Coman M., 2003, Pour une anthropologie des médias, Grenoble, Presses universitaires de Grenoble.

Esquenazi J.-P., 200I, «Télévision et acteurs pluriels du politique », Mots. Les langages du politique, 67, pp. 43-58.

François B. et Neveu É., dirs, 1999, Espaces publics mosaïques. Acteurs, arènes et rhétoriques, des débats publics contemporains, Rennes, Presses universitaires de Rennes.

Genette G., 1972, Figures III, Paris, Éd. Le Seuil.

Gerstlé J., Duhamel O., Davis D. K., 1992, « La couverture télévisée des campagnes présidentielles. L'élection de 1988 aux États-Unis et en France », Pouvoirs, 63, pp. 53-69.

Hanot M., 2002, Télévision : réalité ou réalisme? Introduction à l'analyse sémio-pragmatique des discours télévisuels, Bruxelles/Paris, De Boeck Université/INA.

Jost Fr., 200I, La télévision du quotidien. Entre réalité et fiction, Bruxelles/Paris, De Boeck Université/INA.

— 1999, Introduction à l'analyse de la télévision, Paris, Ellipses.

Le Foulgoc A., 2003, « 1990-2002 : une décennie de politique française à la télévision. Du politique au divertissement », Réseaux, | | 8, pp. 23-63.

Le Grignou B., Neveu é., 1993, « Intimités publiques. Les dynamiques de la politique à la télévision », Revue française de science politique, vol. 43, 6, pp. 940-969.

Lochard G., Soulages J-Cl., 1998, La communication télévisuelle, Paris, A. Colin.

Mehl D., 2002, « La télévision relationnelle », Cahiers internationaux de sociologie, vol. I 12 , pp. 63-95.

— 2008, « La télévision de l'intimité », Le Temps des médias, 10, pp. 265-279.

Metz C., 197I, Essai sur la signification au cinéma,Tome I, Paris, Klincksieck.

Meunier J.-.P., Peraya D., 1993, Introduction aux théories de la communication, Bruxelles, De Boeck Université, 2004.

Miège B., 1989, La société conquise par la communication, Grenoble, Presses universitaires de Grenoble.

Neveu É., 1991 - 1992, « Les émissions politiques à la télévision :l'impossible vulgarisation? 》, Quaderni, 16, pp. 83-99.

— 1997, « Des questions « jamais entendues ». Crise et renouvellement du journalisme politique à la télévision », Politix, vol. 10, 37, pp. 25-56.

— 2003, « De l'art (et du coût) d'éviter la politique. La démocratie du talk-show version française », Réseaux, I| 8, pp. 95- I 34.

Rouquette S., 2002, Vie et mort des débats télévisés, 1958-2000, Bruxelles/Paris, De Boeck Université/INA.

Sepulchre S., 2005, « Analyse des programmes télévisuels de la Communauté française de Belgique », Recherches en communication, 24, pp. 49-66. 
Strömback J., Dimitrova D.V., 2006, « Political and Media Systems Matter : A Comparison of Election News Covarage in Sweden and the United States », The Harvard International Journal of Press/Politics, vol. I I, I31, pp. I31 - 147. 